\title{
Neutralizing efficacy of vaccines against the SARS-CoV-2 Mu variant
}

\author{
Kei Miyakawa ${ }^{1 *}$, Sundararaj Stanleyraj Jeremiah ${ }^{1}$, Hideaki Kato ${ }^{2}$, Akihide Ryo ${ }^{1 *}$ \\ ${ }^{1}$ Department of Microbiology, Yokohama City University School of Medicine, Yokohama, \\ Japan \\ 2 Infection Prevention and Control Department, Yokohama City University Hospital, \\ Yokohama, Japan
}

*Corresponding authors

Kei Miyakawa (keim@yokohama-cu.ac.jp)

Akihide Ryo (aryo@yokohama-cu.ac.jp)

Department of Microbiology, Yokohama City University School of Medicine

3-9 Fuku-ura, Kanazawa, Yokohama 236-0004, Japan 


\begin{abstract}
The rise of mutant strains of SARS-CoV-2 poses an additional problem to the existing pandemic of COVID-19. There are rising concerns about the $\mathrm{Mu}$ variant which can escape humoral immunity acquired from infections from previous strains or vaccines. We examined the neutralizing efficacy of the BNT162b2 mRNA vaccine against the Mu variant and report that the vaccine has $76 \%$ neutralizing effectiveness against the $\mathrm{Mu}$ compared to $96 \%$ with the original strain. We also show that $\mathrm{Mu}$, similar to the Delta variant, causes cell-to-cell fusion which can be an additional factor for the variant to escape vaccine-mediated humoral immunity. Despite the rise in vaccine escape strains, the vaccine still possesses adequate ability to neutralize majority of the mutants.
\end{abstract}

\title{
Main text
}

The rapid and nearly unrestricted global spread of COVID-19 has ensued the evolution of various mutants of SARS-CoV-2. With vaccines being the principal effective modality to curtail the pandemic, we are left with no option than to use them and brace for the rise of immune escape mutants that can evolve due to the selection pressure exerted. Based on clinical and epidemiological significance, the WHO has identified four variants of concern (VOC); Alpha, Beta, Gamma and Delta, and five variants of interest (VOI); Eta, Iota, Kappa, Lambda and $\mathrm{Mu}$ [1]. Despite Delta being the principal mutant responsible for majority of the infections at present, the new kid on the block; $\mathrm{Mu}$ has caused a significant amount of commotion due to its higher propensity for immune escape [2]. In this background, we wanted to evaluate the significance of this alarm raised by Mu with respect to the efficacy of vaccine derived neutralizing antibodies $(\mathrm{nAb})$ and that of the dual antibody cocktail therapy against this variant.

We performed the VLP (virus-like particle)-based rapid neutralization test (hiVNT) [3, 4] on post-vaccination sera collected from individuals one week after administration of the second dose of the BNT162b2 mRNA vaccine. The serum dilution factor that inhibits VLP entry by half $\left(\mathrm{NT}_{50}\right)$ was assessed to demonstrate the neutralizing activity of these sera against the variants $\mathrm{Mu}$, Alpha, Beta, Gamma, Delta and Lambda. The median neutralizing efficacy for all the variants was above the effective threshold $\left(\mathrm{NT}_{50}=20\right)$ in all the tested sera suggesting that the vaccine derived nAbs can neutralize majority of all the variants including $\mathrm{Mu}$ (Figure 1A, S1A). Previously infected individuals showed prominently higher $\mathrm{NT}_{50}$ values for all variants after vaccination (Figure S2). 
We then wanted to grade the efficacy of the $\mathrm{nAb}$ response in post vaccinated sera against the different variants. For this we used pvNT $_{50}$ (Serum dilution factor that inhibits HIV-based pseudovirus infection by half) of 40 as the lower threshold and pvNT 50 of 200 as the higher threshold. This is based on a recent report that the $\mathrm{pvNT}_{50}$ in sera of individuals with vaccinebreakthrough infections was around 200 [5]. Samples that fell below the lower threshold were deemed negative for neutralizing activity, while those in between the lower and higher threshold were considered to possess weak neutralizing activity and those above the higher threshold reflected strong neutralizing activity (Figure 1B, S1B). Strong neutralization of all variants was observed in majority of the sera ranging from the highest of $96 \%$ in D614G to the lowest $72 \%$ in Beta variant. Mu showed a pattern similar to Beta with $76 \%$ of the samples strongly neutralized. The proportion of sera that did not neutralize was much lower when compared to those possessing neutralizing efficacy for each variant. The highest occurrence of nAb escape (including weak and non-neutralizing activity) was noted with Beta (28\%) followed by $\mathrm{Mu}(24 \%)$.

We then evaluated the efficacy of the dual antibody cocktail against these variants and found that all the tested variants including $\mathrm{Mu}$ were neutralized by at least one of the two antibodies in the cocktail (Figure 1C). We further demonstrate that the $\mathrm{Mu}$ variant can also cause cellcell fusion (Figure S3) like Delta variant, which is highly likely to promote the viral resistance to nAbs [6].

With rise of the $\mathrm{Mu}$ variant, there has been a panic concerning the efficacy of the currently available vaccines. Our results show that the vaccine derived nAbs and the antibody cocktail still possess adequate neutralization efficacy against this variant. We observed this effect in sera of vaccine recipients shortly after the receipt of the second dose when the nAbs are supposed to be at peak levels. As vaccine derived nAbs can wane over time, follow-up studies are needed to assess the persistence of $\mathrm{nAbs}$ against the $\mathrm{Mu}$ variant. By possessing the property of cell fusion like the Delta variant, but with a higher proportion of escape from the vaccine than the latter, $\mathrm{Mu}$ could especially be a problem if it would replace the Delta as the most predominant variant. Despite this, the current vaccines and antibody cocktail would still work for the majority, albeit at a slightly lowered efficacy than currently observed with the Delta variant. 


\section{References}

1. WHO. Tracking SARS-CoV-2 variants. https://www.who.int/en/activities/trackingSARS-CoV-2-variants/

2. Messali S, Bertelli A, Campisi G, et al. A cluster of the new SARS-CoV-2 B.1.621 lineage in Italy and sensitivity of the viral isolate to the BNT162b2 vaccine. J Med Virol 2021.

3. Miyakawa K, Jeremiah SS, Ohtake N, et al. Rapid quantitative screening assay for SARS-CoV-2 neutralizing antibodies using HiBiT-tagged virus-like particles. J Mol Cell Biol 2020; 12:987-90.

4. Miyakawa K, Jeremiah SS, Kato H, et al. Rapid detection of neutralizing antibodies to SARS-CoV-2 variants in post-vaccination sera. J Mol Cell Biol 2021.

5. Bergwerk M, Gonen T, Lustig Y, et al. Covid-19 Breakthrough Infections in Vaccinated Health Care Workers. N Engl J Med 2021.

6. Mlcochova P, Kemp S, Dhar MS, et al. SARS-CoV-2 B.1.617.2 Delta variant replication and immune evasion. Nature 2021.

Acknowledgement We acknowledge all medical staff involved in the study. We thank Kenji Yoshihara and Kazuo Horikawa for their technical assistance. This study was supported by AMED grants (JP20he0522001, JP21fk0108104) to AR.

Conflict of interests Authors have no conflicts of interest directly relevant to the content of this article.

Author contributions KM designed and performed the research, analyzed the data, and wrote the manuscript; SSJ analyzed the data and wrote the manuscript; HK collected the specimens; AR directed the research, analyzed the data, and wrote the manuscript. 


\section{Figure}

A

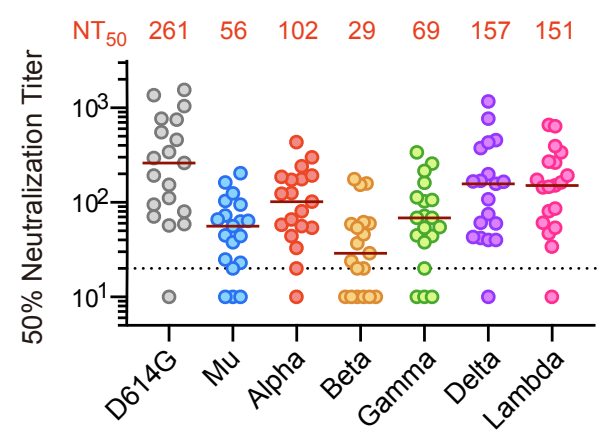

B

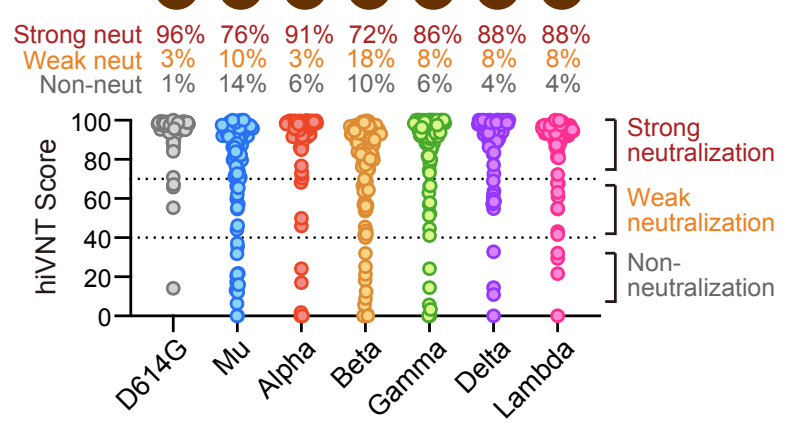

C
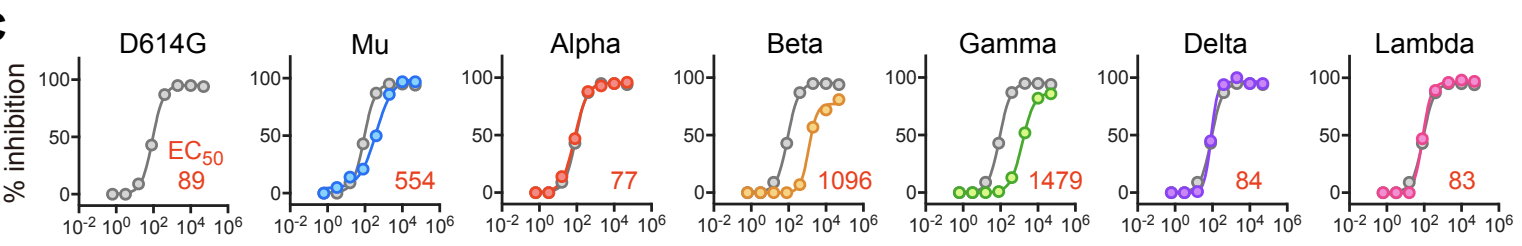

Casirivimab $(\mathrm{ng} / \mathrm{mL})$
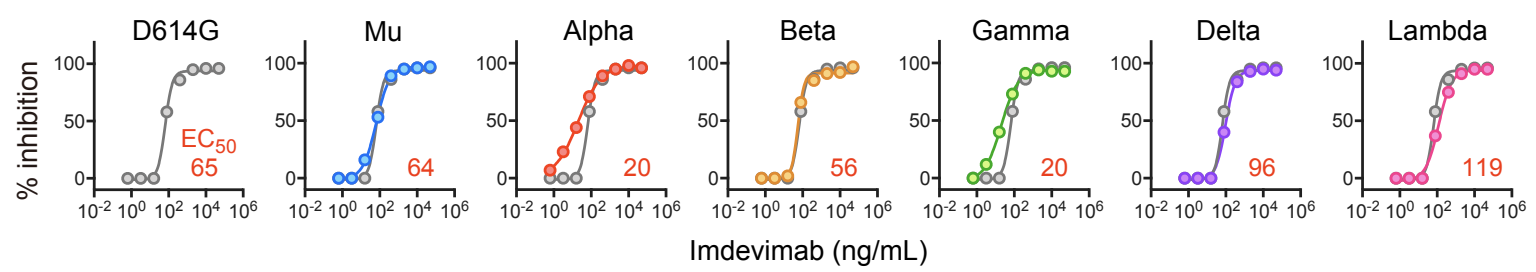

Figure 1. Neutralization of SARS-CoV-2 Mu variant by vaccine sera and monoclonal antibodies

(A) Neutralizing activity of BNT162b2 post-vaccination sera (1 week after second dose) against each variant $(\mathrm{n}=19)$. Serum dilutions showing $50 \%$ inhibition of infection $\left(\mathrm{NT}_{50}\right)$ were determined by quantitative hiVNT. The dotted line indicates the cut-off threshold of this assay $\left(\mathrm{NT}_{50}=20\right)$. The red lines indicate the median $\mathrm{NT}_{50}$ and the values of which are displayed as red numerals above each parameter in the graph. Note that this assay correlates well with the assay using the authentic virus (see Figure S1A).

(B) Positive rates for neutralizing antibody determined by qualitative hiVNT. BNT162b2 post-vaccination sera ( 1 week after second dose) were used $(n=105)$. The percentage of inhibition of viral infection by 20 -fold dilution of serum is shown in the scatter plot as hiVNT score. hiVNT score below 40 (equivalent to $\mathrm{pvNT}_{50}<50$ ) is defined as nAb-negative serum, $40-70$ (equivalent to $\mathrm{pvNT}_{50}>50$ but $<200$ ) as weakly neutralizing serum, and above 70 (equivalent to $\mathrm{pvNT}_{50}>200$ ) as strongly neutralizing serum. The percentage of each serum 
is shown in the pie chart. Note that the qualitative hiVNT provides semi-quantitative results (see Figure S2B for the description of the accuracy of this definition).

(C) Neutralization curve of each mutant strain by two monoclonal antibodies (Casirivimab and Imdevimab). The red numbers indicate the $50 \%$ effective concentration $\left(\mathrm{EC}_{50}, \mathrm{ng} / \mathrm{mL}\right)$. For comparison, the neutralization curve for each mutant is superimposed on that of the D614G control (gray line). Since these $\mathrm{nAb}$ medicines are treated as a cocktail, they are considered effective if the $\mathrm{EC}_{50}$ of either antibody is equivalent to or lower than that of the D614G control. 


\section{Supplementary Appendix}
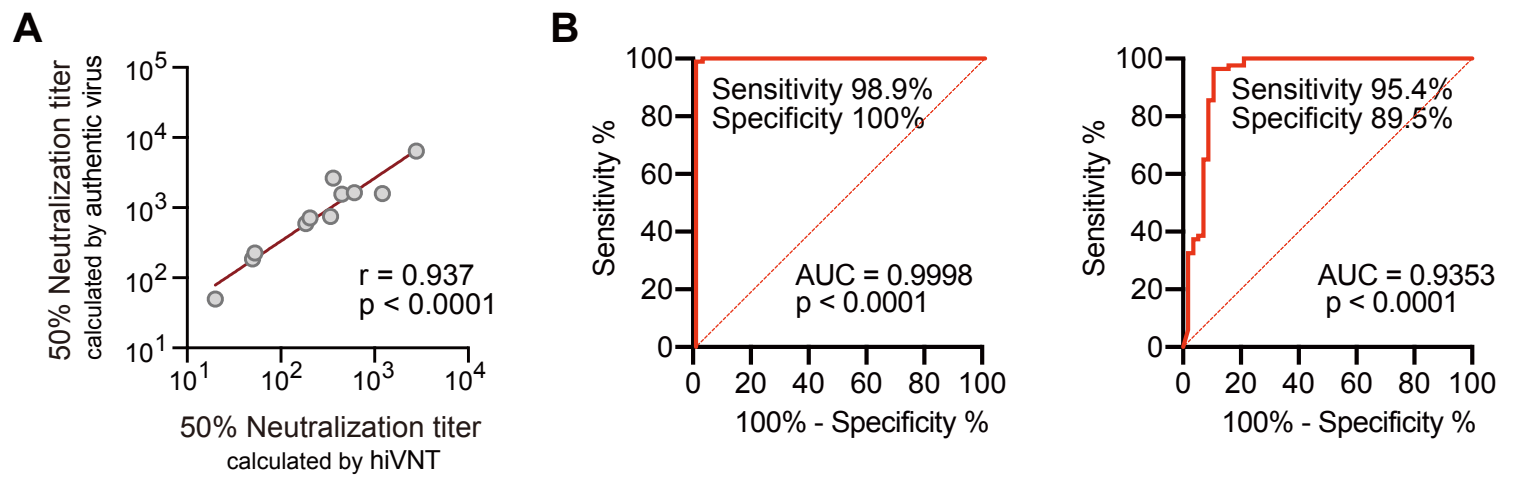

\section{Supplementary Figure S1. Accuracy of hiVNT used in this study}

(A) Correlation between the quantitative hiVNT and the standard neutralization assay using authentic virus. Eleven sera were plotted as serum dilutions showing $50 \%$ inhibition of infection by each method.

(B) Accuracy of qualitative hiVNT scoring and neutralization activity. On the left panel shows the receiver operating characteristic (ROC) curve when a hiVNT score of 40 is defined as a $\mathrm{nAb}$ negative $\left(\mathrm{pvNT}_{50}<50\right)$ in the pseudovirus neutralization test. On the right panel shows the ROC curve when a hiVNT score of 70 or higher is defined as a serum with strong neutralizing activity $\left(\mathrm{NT}_{50}>200\right)$. Although the qualitative hiVNT method measures only 20 -fold dilution of serum, it still provides semi-quantitative results [note that Area under curve (AUC) $>0.90$ in both cases]. 


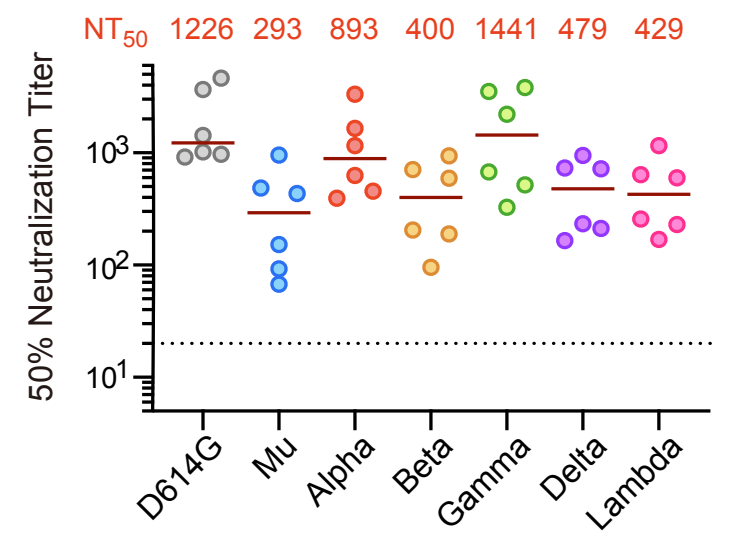

Supplementary Figure S2. Neutralization of SARS-CoV-2 Mu variant by vaccine sera in previously infected individuals

Neutralizing activity of sera after BNT162b2 vaccination (1 week after the second dose) against each variant in previously infected individuals $(\mathrm{n}=6)$. The red line and numbers in the graph indicate the median values. Serum dilutions that inhibit infection by $50 \%$ were determined by quantitative hiVNT. The dotted line indicates the threshold value in this assay. 


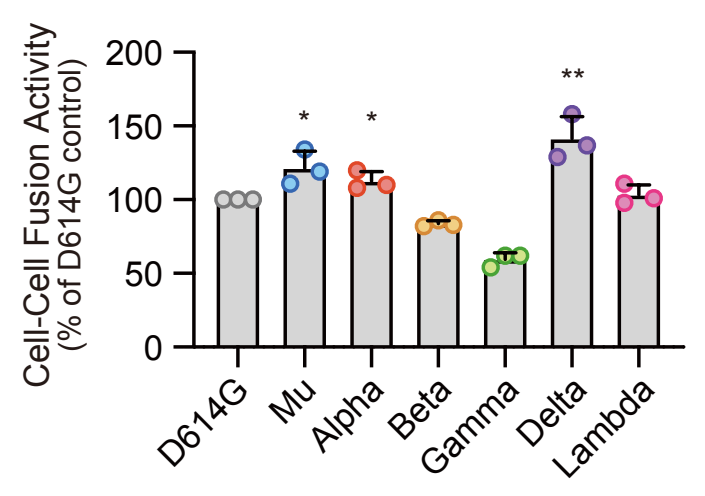

\section{Supplementary Figure S3. Cell fusion activity of SARS-CoV-2 variant}

Cell-to-cell fusion activity of indicated variants. Assay was carried out using a split luciferase system. HEK293-donor cells (expressing SARS-CoV-2 spike and HiBiT) and HEK293acceptor cells (expressing LgBiT and ACE2) were cocultured in 1:1 ratio. After 3 hours, the luciferase activity derived from cell fusion was measured. $* P<0.05, * * P<0.01$. Higher luciferase signal denotes higher occurrence of cell-to-cell fusion. 


\section{Supplementary Materials}

\section{Ethics statement}

This study was approved by Yokohama City University Certified Institutional Review Board (Reference No. B210300001), and the protocols used in the study were approved by the ethics committee. Written informed consent was obtained from all the participants.

\section{Rapid neutralization test (hiVNT)}

hiVNT was performed as previously described [3, 4]. Briefly, target cells seeded in 96-well plates were inoculated with $50 \mu \mathrm{L}$ of HiBiT-tagged virus-like particles (hiVLPs) containing diluted serum (1:20-1:43,740 dilution for quantitative assay; 1:20 dilution for qualitative assay). At 3 hours after inoculation, intracellular luciferase activity is measured. The dilution factor of serum that resulted in a 50\% reduction in luminescence compared with the nonserum control was set as the $\mathrm{NT}_{50}$. We calculated $\mathrm{NT}_{50}$ using the curve-fitting tool (ImageJ, $\mathrm{NIH}$ ). When serum had no observable neutralizing activity to interpolate $\mathrm{NT}_{50}$, it was assigned an $\mathrm{NT}_{50}$ of 10 . All samples were assayed in duplicate.

In qualitative assay, the hiVNT score (percentage of luminescence signal inhibition) was calculated as follows:

$$
\frac{\text { RLU (without serum) }- \text { RLU (with serum) }}{\text { RLU (without serum) }- \text { RLU (blank) }} \times 100
$$

Alternatively, cells were inoculated with $50 \mu \mathrm{L}$ of hiVLPs containing diluted antibody (final concentration of $0.64-50,000 \mathrm{ng} / \mathrm{mL}$ ). The concentration of antibody that resulted in a $50 \%$ reduction in luminescence compared with the non-antibody control was set as $\mathrm{EC}_{50}$. 